\title{
Avaliação do Sucesso do Desmame da Ventilação Mecânica*
}

\section{Assessment of Success in Weaning from Mechanical Ventilation}

\author{
Edna Estelita Costa Freitas ${ }^{1}$, Cid Marcos Nascimento David ${ }^{2}$
}

\section{RESUMO}

JUSTIFICATIVA E OBJETIVOS: O desmame dos pacientes sob ventilação mecânica (VM) é uma das etapas críticas da assistência ventilatória em terapia intensiva. Existem vários critérios para a retirada dos pacientes de prótese respiratória. O objetivo deste trabalho foi avaliar se existe um grupo de parâmetros que podem predizer os pacientes que irão desmamar com sucesso da ventilação mecânica.

MÉTODO: Sessenta pacientes foram estudados de forma prospectiva em 24 meses; todos se encontravam em VM por tempo $\geq 48$ horas. Foram monitorados os parâmetros mecânicos específicos para o desmame, os dados clínicos, os valores gasométricos e os resultados laboratoriais. Os pacientes foram divididos em grupos de sucesso e de insucesso, para as análises comparativas. Pela curva ROC observou-se o melhor ponto de corte para as variáveis numéricas avaliadas para o sucesso do desmame.

1. Mestre em Clínica Médica (Terapia Intensiva). Médica do Centro de Tratamento Intensivo (CTI) do HUCFF da UFRJ. Coordenadora do Grupo de Ventilação Mecânica do CTI do HUCFF. Médica Especialista em Terapia Intensiva pela Associação de Medicina Intensiva Brasileira (TE-AMIB).

2. Professor Adjunto da Faculdade de Medicina da UFRJ. Doutor em Ciências Médicas. Chefe do CTI do HUCFF. Presidente do Fundo Brasileiro de Educação e Pesquisa em Medicina Intensiva - FUNDO AMIB. TE-AMIB.

*Recebido da Pós-Graduação de Terapia Intensiva da Universidade Federal do Rio de Janeiro (UFRJ) do Hospital Universitário Clementino Fraga Filho (HUCFF).

- Tese de dissertação do mestrado defendida em 15 de janeiro de 2004.

Apresentado em 19 de junho de 2006

Aceito para publicação em 12 de dezembro de 2006

Endereço para correspondência:

Rua Brigadeiro Trompowski s $/ \mathrm{n}^{\circ}-13^{\circ}$ Andar-CTI - Ilha do Fundão. 21945-560 Rio de Janeiro, RJ

Fone: (21) 2562-2524 - 2562-2496 Fax: 2562-2524.

E-mail: estelita@iis.com.br

(C)Associação de Medicina Intensiva Brasileira, 2006
RESULTADOS: Na análise de regressão logística realizada para avaliar a influência simultânea de todos os fatores: VM $\leq 8$ dias, APACHE II $\leq 16$ e Pimáx (pressão inspiratória máxima) $>(-) 20 \mathrm{cmH}_{2} \mathrm{O}$ foram estatisticamente significativos para predizer o sucesso ao desmame, nessa ordem de capacidade explicativa.

CONCLUSÕES: Os índices avaliados foram adequados na determinação do sucesso do desmame desses pacientes em ventilação mecânica. O APACHE II por ocasião da internação constituiu indicador de gravidade e permitiu maior vigilância do paciente. Monitoração do tempo de VM, a otimização do tratamento no sentido de acelerar o processo de desmame são condutas que visam não só o seu sucesso, mas interferem na evolução e no tempo de internação hospitalar.

Unitermos: Desmame ventilatório, ventilação mecânica.

\section{SUMMARY}

BACKGROUND AND OBJECTIVES: The weaning of patients under mechanical ventilation (MV) is one of the critical stages of respiratory assistance in intensive care. There are several criteria for taking patients out of respiratory prothesis. The aim of this work was to assess if there is a group of parameter which can predict the patients who will succeed in weaning from mechanical ventilation.

METHODS: Sixty patients were studied in a prospective way within 24 months. All of them had been in MV for, time $\geq 48$ hours. The specific mechanical parameters were monitored for the weaning, clinical data, gasometrical values and laboratory results. The patients were divided into both succeeding and unsucceeding groups for comparable analysis. By the ROC curve, it was observed the best cut point for the numerical variables evaluated for the success of the weaning.

RESULTS: In analysis of logistic regression performed to evaluate the simultaneous influence of all the factors: $\mathrm{MV} \leq 8$ days, APACHE II (Acute Physiologic and Chronic Health Evaluation II) $\leq 16$ and Pimax (maximum 
respiratory pression) $>$ (-) $20 \mathrm{cmH}_{2} \mathrm{O}$ were statistically significant to predict the success to weaning, in this order of explainable capacity.

CONCLUSIONS: We could conclude that the indexes evaluated were suitable for the determination of the success in the weaning of those patients in mechanical ventilation. APACHE II because of admition constitutes severity indicator and allows awareness from the patient. MV timing, optimizing the treatment in order to accelerate the process of weaning is conducts that aim not only for the weaning success but also interfere both in the evolution and period of hospital admition.

Key Words: mechanical ventilation, Ventilator weaning

\section{INTRODUÇÃO}

A ventilação mecânica (VM) é a modalidade mais utilizada de suporte de vida na insuficiência respiratória aguda (IRA) ${ }^{1}$.

Esteban e col. ${ }^{2}$ conduziram um estudo transversal, em diversas unidades de terapia intensiva e observaram que a prevalência de pacientes em VM era de $42 \%$ no Brasil e de $39 \%$ nos demais países estudados ${ }^{3,4}$.

Uma vez que a condição determinante da IRA tenhase resolvido, a VM pode ser interrompida. A resolução da lesão pulmonar; entretanto, é um processo lento, assim como a restauração da função muscular respiratória após período em VM. O fato de a lesão pulmonar não estar completamente resolvida ou de a musculatura respiratória não ter recuperado sua capacidade funcional não impedem o início do desmame $^{1,5}$

O desmame é um processo cujo início jamais deve ser adiado, tendo em vista as complicações associadas à VM e aos custos relacionados com esse processo.

Vários fatores preditivos têm sido utilizados para avaliar a capacidade da musculatura respiratória em manter a ventilação espontânea, indicando maior ou menor taxa de sucesso do desmame. Ao se avaliar a possibilidade de sucesso do desmame deve-se levar em consideração a existência de um equilíbrio entre a carga imposta à bomba muscular respiratória, sua capacidade de sustentar essa carga e os mecanismos envolvidos no controle da ventilação. Atualmente, usam-se medidas que traduzem justamente esses fatores. A apreciação conjunta dessas medidas ou índices pode oferecer uma noção acerca do sucesso dessa intervenção ${ }^{3,6}$.

Os objetivos da nossa pesquisa foram principalmente determinar quais os parâmetros medidos antes de iniciar o desmame em pacientes sob ventilação mecânica que podem predizer o sucesso do desmame. Os objetivos secundários foram correlacionar à evolução do desmame com a presença de complicações pulmonares, renais, utilização de ventilação mecânica não-invasiva (VMNI) intermitente após a extubação e, em caso de insucesso do desmame, a realização de traqueostomia (TQT).

Como a insuficiência respiratória é uma resposta à integração do sistema cardiopulmonar, as medidas que avaliam somente a função pulmonar são insuficientes para orientar as decisões quanto ao desmame. Tal fato demonstra que, em alguns pacientes, outras condições, que não as ventilatórias, sejam responsáveis pelo insucesso do desmame ${ }^{7-9}$. O melhor entendimento de parâmetros monitorados evolutivamente durante a ventilação mecânica e a realização do desmame, incluindo todos os critérios clínicos e fisiológicos estabelecidos, mediante estudos acurados é primordial para a identificação dos fatores que realmente definem o sucesso da interrupção da VM.

\section{MÉTODO}

Estudo clínico prospectivo observacional com coleta consecutiva de pacientes em VM por período igual ou maior que 48 horas internados no centro de tratamento intensivo (CTI) da Casa de Saúde São José, Rio de Janeiro, RJ, que apresentaram os critérios para desmame. O estudo foi realizado no período de 1 de julho de 2001 a 1 de julho de 2003, quando completou 60 pacientes. Os dados foram colocados na ficha de monitorização em planilha eletrônica (Excel).

Os critérios de inclusão foram pacientes em VM, por no mínimo, 48 horas ou mais. Os pacientes com critérios de desmame: resolução ou controle do motivo que o levou à ventilação mecânica; estabilidade hemodinâmica; relação pressão parcial arterial de oxigênio e fração inspirada de oxigênio $\left(\mathrm{PaO}_{2} / \mathrm{F}_{1} \mathrm{O}_{2}\right)$ $\geq 200$; pressão expiratória final positiva (PEEP) $\leq 8$ $\mathrm{cmH}_{2} \mathrm{O}$, ou desmame decidido pelo médico-assistente do paciente (que coincidentemente obedeceu aos critérios estabelecidos pelo trabalho).

Os critérios de exclusão são citados no fluxograma do protocolo 1. 
Fluxograma do Protocolo 1

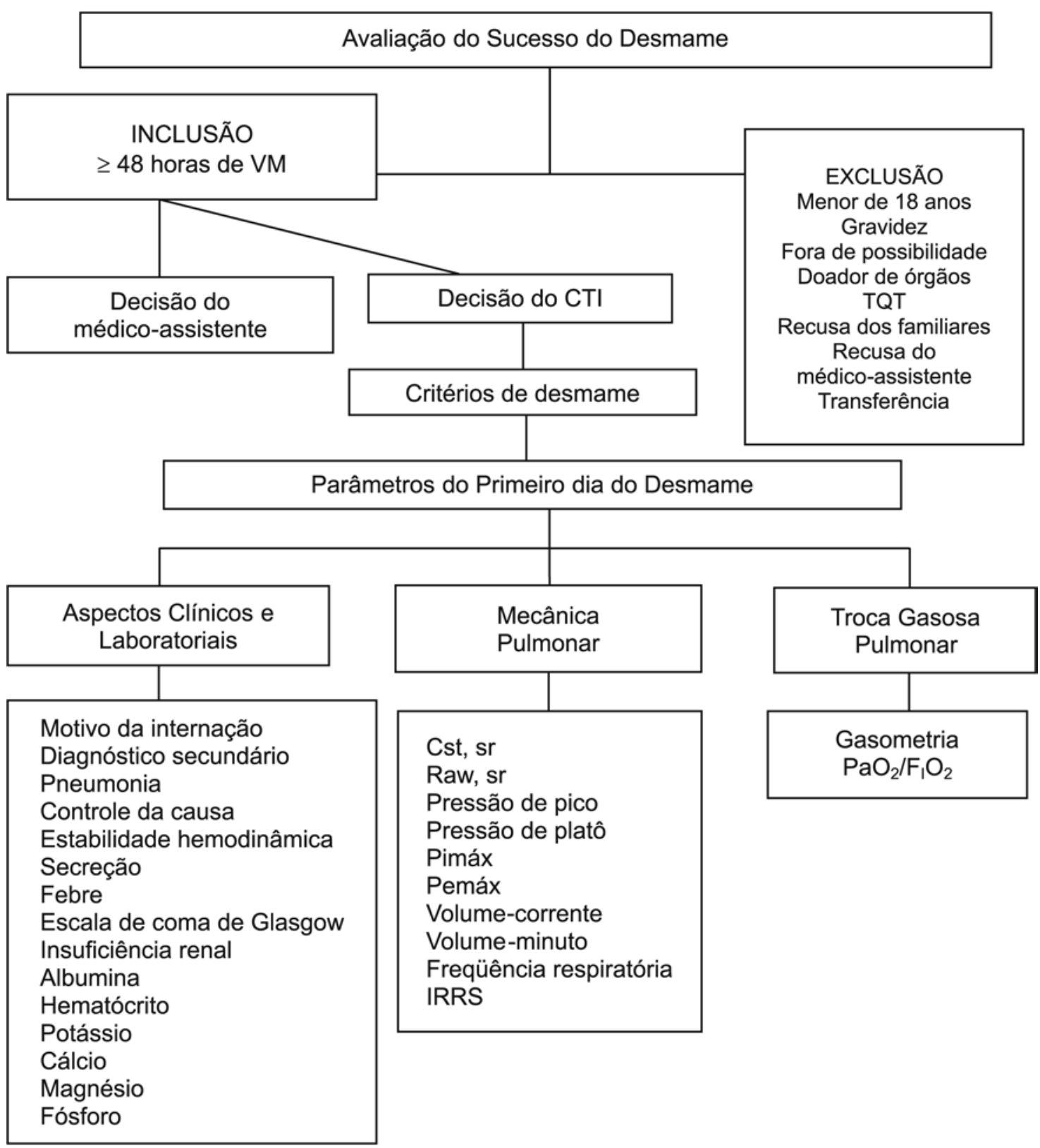

Assim que o paciente foi incluído no trabalho foram anotados os seguintes parâmetros: clínicos, mecânicos pulmonares, gasométricos e laboratoriais.

A avaliação foi realizada de acordo com os parâmetros descritos, medidos antes de iniciar o desmame do grupo de pacientes incluídos para determinar quais, ou se algum, desses parâmetros foi adequado para predizer o sucesso do desmame.

O método de desmame foram peça $T$ por 30 minutos ou PSV diminuído gradualmente em $2 \mathrm{cmH}_{2} \mathrm{O}$ a cada
2 horas, até um nível de $7 \mathrm{cmH}_{2} \mathrm{O}$. Foi considerado sucesso do desmame quando o paciente permaneceu 48 horas ou mais fora de prótese ventilatória.

A análise estatística foi realizada visando verificar se existia diferença significativa nas variáveis estudadas entre os dois grupos de sucesso(S) e insucesso(I). Para comparação de proporções, foi utilizado o teste de Qui-quadrado $\left(\chi^{2}\right)$ ou o teste Exato de Fisher; a comparação das variáveis quantitativas entre dois grupos foi analisada pelo teste de Mann-Whitney. O 
critério de determinação significativo adotado foi o nível de $5 \%$.

A análise estatística foi processada pelo software estatístico SAS ${ }^{\circledR}$ System.

A curva ROC (Receiver Operator Characteristic) foi usada para escolher o melhor ponto de corte (ponto ótimo). A curva ROC foi ajustada pelo algoritmo adaptado de Graphical Methods for Data Analysis (John Chambers et al., Wadsworth and Brooks, 1983).

A análise de regressão logística foi realizada para avaliar a influência simultânea dos fatores prognósticos sobre o sucesso no tratamento de desmame da população em estudo. Os fatores considerados para a regressão foram os da Análise Univariada.

O processo de seleção dos fatores foi o de stepwise, em que se selecionou o menor subgrupo de variáveis independentes que melhor explicou o sucesso. As variáveis foram selecionadas à medida que acrescentaram informação discriminatória que as anteriores não possuam.

Os parâmetros de cada fator foram estimados pelo método de máxima verossimilhança.

Projeto de pesquisa submetido ao Comitê de Ética e Pesquisa da Universidade Federal do Rio de Janeiro aprovado em 27/11/2001 e com termo de consentimento livre e esclarecido.

\section{RESULTADOS}

Foram estudados sessenta pacientes. Nenhum foi excluído.

Os respiradores utilizados foram Bird 8400 e Servo 300. Os dados foram coletados no modo assisto-controlado no momento em que foi decidido o desmame.

A tabela 1 fornece a média, o desvio-padrão (DP), o mínimo e o máximo das variáveis numéricas para o total da amostra.

Dos 60 pacientes, 35 pertenciam ao sexo masculino e 25 ao sexo feminino. Quinze pacientes evoluíram para óbito e 45 obtiveram alta do CTI.

Grande parte do motivo de internação no CTI foi o pósoperatório, seguindo-se por pneumonia e sepse. (Figura 1), sendo a maior parte dos pós-operatórios de cirurgias abdominais. Independentemente do motivo que levou o paciente à ventilação mecânica esse poderia apresentar pneumonia antes da sua internação no CTI, fato que ocorreu com 25 pacientes. Entre os motivos que levaram o paciente a VM, os principais foram a hipoxemia e a instabilidade hemodinâmica (choque circulatório com PAM $<70 \mathrm{mmHg}$ com necessidade do uso de agentes vasoativos).
Tabela 1 - Análise Descritiva Geral

\begin{tabular}{|c|c|c|c|c|}
\hline Variáveis & $\begin{array}{l}\mathrm{N}^{\circ} \\
\mathrm{Pac} .\end{array}$ & Média \pm DP & Mínimo & Máximo \\
\hline Idade (anos) & 60 & $69,52 \pm 17,16$ & 18 & 97 \\
\hline $\begin{array}{l}\text { Tempo de interna- } \\
\text { ção (dias) }\end{array}$ & 60 & $24,63 \pm 18,40$ & 5 & 100 \\
\hline APACHE II & 60 & $16,52 \pm 5,72$ & 6 & 30 \\
\hline $\begin{array}{l}\text { Ventilação mecâ- } \\
\text { nica (dias) }\end{array}$ & 60 & $18,93 \pm 18,39$ & 4 & 100 \\
\hline $\begin{array}{l}V \mathrm{~V} / \mathrm{d} \text { e s } \mathrm{m} \text { a } \mathrm{m} \text { e } \\
\text { (dias) }\end{array}$ & 60 & $9,78 \pm 5,24$ & 4 & 24 \\
\hline Desmame (dias) & 60 & $1,12 \pm 0,49$ & 1 & 4 \\
\hline ECG & 60 & $10,77 \pm 0,91$ & 5 & 11 \\
\hline Pimáx $\left(\mathrm{cmH}_{2} \mathrm{O}\right)$ & 60 & $32,20 \pm 15,21$ & 10 & 98 \\
\hline Pemáx $\left(\mathrm{cmH}_{2} \mathrm{O}\right)$ & 60 & $32,90 \pm 15,67$ & 10 & 76 \\
\hline IRRS (irpm/l) & 60 & $80,52 \pm 42,19$ & 19 & 238 \\
\hline Cst $\left(\mathrm{mL} / \mathrm{cmH}_{2} \mathrm{O}\right)$ & 60 & $36,70 \pm 13,55$ & 15 & 70 \\
\hline $\mathrm{Rwa}\left(\mathrm{cmH}_{2} \mathrm{O} / \mathrm{L} / \mathrm{s}\right)$ & 60 & $10,27 \pm 3,62$ & 4 & 20 \\
\hline $\mathrm{P}_{\mathrm{Pl}}\left(\mathrm{cmH}_{2} \mathrm{O}\right)$ & 60 & $24,28 \pm 6,23$ & 10 & 38 \\
\hline $\mathrm{P}_{\mathrm{PLAT}}\left(\mathrm{cmH}_{2} \mathrm{O}\right)$ & 60 & $18,82 \pm 4,93$ & 8 & 30 \\
\hline PEEP $\left(\mathrm{cmH}_{2} \mathrm{O}\right)$ & 60 & $5,08 \pm 0,74$ & 3 & 10 \\
\hline $\begin{array}{l}\mathrm{P} \text { i m á } x / \mathrm{P}_{\mathrm{PLAT}} \\
\left(\mathrm{cmH}_{2} \mathrm{O}\right)\end{array}$ & 60 & $1,90 \pm 1,36$ & 0,53 & 9,8 \\
\hline $\mathrm{VC}(\mathrm{mL})$ & 60 & $417,50 \pm 132,79$ & 160 & 714 \\
\hline $\mathrm{Vm}(\mathrm{L} / \mathrm{min})$ & 60 & $11,22 \pm 5,29$ & 4,6 & 29,9 \\
\hline FR (irpm) & 60 & $27,93 \pm 8,71$ & 13 & 50 \\
\hline $\mathrm{Ht}(\mathrm{g} \%)$ & 60 & $31,04 \pm 4,55$ & 18,8 & 41 \\
\hline $\mathrm{K}^{+}(\mathrm{mEq})$ & 60 & $3,76 \pm 0,57$ & 2,4 & 5,3 \\
\hline $\mathrm{Mg}^{+}(\mathrm{mmol})$ & 60 & $2,19 \pm 0,52$ & 1,2 & 3,9 \\
\hline $\mathrm{Ca}^{++}(\mathrm{mmol})$ & 60 & $8,31 \pm 0,82$ & 6 & 9,7 \\
\hline $\mathrm{PO}_{4^{-}}(\mathrm{mmol})$ & 60 & $2,88 \pm 0,96$ & 1,1 & 5,1 \\
\hline $\mathrm{pH}$ & 60 & $7,44 \pm 0,05$ & 7,31 & 7,54 \\
\hline $\mathrm{PaO}_{2}(\mathrm{mmHg})$ & 60 & $101,17 \pm 25,29$ & 64,7 & 168,7 \\
\hline $\mathrm{PaCO}_{2}(\mathrm{mmHg})$ & 60 & $36,75 \pm 5,62$ & 23,7 & 49,8 \\
\hline $\mathrm{HCO}_{3}^{-}(\mathrm{mmol})$ & 60 & $25,48 \pm 4,11$ & 18,1 & 38,8 \\
\hline $\mathrm{F}_{1} \mathrm{O}_{2}$ & 60 & $0,322 \pm 0,047$ & 0,210 & 0,400 \\
\hline $\mathrm{PaO}_{2} / \mathrm{F}_{1} \mathrm{O}_{2}(\mathrm{mmHg})$ & 60 & $314,20 \pm 65,29$ & 202 & 483 \\
\hline $\mathrm{pH}^{(*)}$ & 10 & $7,28 \pm 0,14$ & 7,1 & 7,44 \\
\hline $\mathrm{PaO}_{2}^{\left({ }^{()}\right.}(\mathrm{mmHg})$ & 10 & $109,73 \pm 68,12$ & 48 & 271,9 \\
\hline $\mathrm{PaCO}_{2}^{(*)}(\mathrm{mmHg})$ & 10 & $51,29 \pm 19,69$ & 27,6 & 101,9 \\
\hline Lactato(*) & 10 & $21,96 \pm 6,53$ & 12,7 & 34 \\
\hline $\mathrm{PSV}\left(\mathrm{cmH}_{2} \mathrm{O}\right)$ & 30 & $11,83 \pm 3,75$ & 8 & 20 \\
\hline PEEP $\left(\mathrm{cmH}_{2} \mathrm{O}\right)$ & 31 & $5,58 \pm 1,31$ & 5 & 10 \\
\hline TQT/TOT (dias) & 19 & $16,11 \pm 5,53$ & 8 & 26 \\
\hline VMNI E $\left(\mathrm{cmH}_{2} \mathrm{O}\right)$ & 31 & $7,26 \pm 1,83$ & 5 & 12 \\
\hline VMNI I $\left(\mathrm{cmH}_{2} \mathrm{O}\right)$ & 31 & $14,39 \pm 2,80$ & 8 & 18 \\
\hline DPAVM (dias) & 18 & $14,00 \pm 6,85$ & 4 & 28 \\
\hline
\end{tabular}

() Resultados encontrados nos pacientes com insucesso por fadiga; TQT/TOTdia de intubação traqueal que foi realizada a TQT; VMNI E- pressão expiratória utilizada na ventilação mecânica não-invasiva; VMNI I-pressão inspiratória utilizada na ventilação não invasiva; DPAVM-dia de VM que apresentou PAVM. 


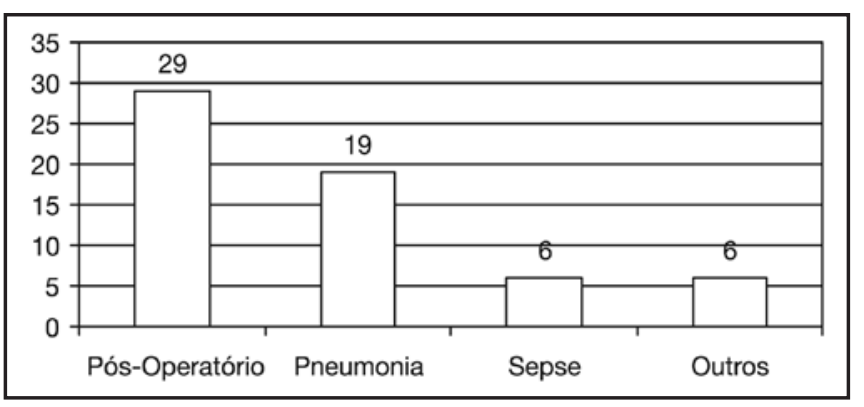

Figura 1 - Motivo de Internação no CTI

Todos os pacientes encontravam-se estáveis hemodinamicamente e a maioria sem secreção traqueobrônquica, sem febre e com resolução ou controle do motivo que o levou à prótese, apesar da interferência, por vezes, do médico assistente no desmame.

Dos 60 pacientes, 34 evoluíram com sucesso e 26 com insucesso. No grupo insucesso houve falha em 10 por fadiga e 16 por hipoxemia. Nos 10 que apresentaram fadiga, definida como hipoxemia associada à hipercapnia e acidose respiratória com $\mathrm{pH}<7,2$, nove tiveram alteração da consciência. A principal causa de hipoxemia sem hipercapnia foi o edema agudo de pulmão (EAP).

O método de desmame, peça T e PSV, foi por coincidência em número igual nos dois grupos.

Observou-se que o grupo com sucesso no desmame da VM apresentou dias de internação $(16,16 \pm$ $11,24$ versus $35,69 \pm 20,19 ; p=0,0001)$, APACHE II $(14,97 \pm 4,71$ versus $18,54 \pm 6,36 ; p=0,014)$, dias de VM $(9,32 \pm 5,92$ versus $31,50 \pm 21,49 ; p$ $=0,0001$ ), dias de VM em que foi realizado o desmame (dias de VM/desmame 8,38 \pm 4,41 versus $11,62 \pm 5,73 ; p=0,020)$, índice de respiração rápida e superficial (IRRS 66,41 \pm 29,03 versus 98,96 \pm $49,64 ; p=0,004)$, resistência de via aérea do sistema respiratório (Raw, sr 9,26 $\pm 3,01$ versus 11,58 \pm $3,97 ; p=0,031)$ e freqüência respiratória $(25,12 \pm$ $7,05$ versus $31,62 \pm 9,40 ; p=0,005)$ significativamente menores do que o grupo com insucesso. $O$ grupo com sucesso no desmame da VM apresentou escala de coma de Glasgow, sendo considerado o máximo no paciente intubado no valor de $11(10,97 \pm 0,17$ versus $10,50 \pm 1,33 ; p=0,035)$, Pimáx $(36,15 \pm 15,47$ versus $27,04 \pm 13,46 ; p=$ 0,013 ), pressão expiratória máxima (Pemáx 37,15 $\pm 14,32$ versus $27,35 \pm 15,87 ; p=0,004)$, com- placência estática do sistema respiratório (Cst, sr $40,38 \pm 12,76$ versus $31,88 \pm 13,26 ; p=0,006)$, hematócrito $(32,13 \pm 3,86$ versus $29,62 \pm 5,05 ; p=$ $0,025)$ significativamente maiores do que o grupo com insucesso.

A tabela 2 fornece a média, o desvio-padrão (DP), o mínimo e o máximo das variáveis numéricas segundo o grupo. A análise estatística foi realizada pelo teste de Mann-Whitney.

Observou-se que a proporção de homens no grupo com sucesso $(64,7 \%)$ não diferiu significativamente do grupo com insucesso (50\%), com $p=0,25$, a proporção de óbito no grupo com sucesso $(5,9 \%)$ foi significativamente menor do que no grupo com insucesso $(50 \%)$, com $p<0,001$, a proporção de motivos da internação no grupo com sucesso não diferiu significativamente do grupo com insucesso (com $p=0,28)$ a proporção de pneumonia pré-internação no grupo com sucesso $(55,9 \%)$ foi significativamente maior do que no grupo com insucesso $(23,1 \%)$, com $p=0,011$, a proporção de instabilidade hemodinâmica antes do desmame no grupo com sucesso $(40,6 \%)$ não diferiu significativamente do grupo com insucesso $(65,2 \%)$, com $p=$ 0,072, a proporção de método PSV no grupo com sucesso $(47,1 \%)$ não diferiu significativamente do grupo com insucesso (53,8\%), com $p=0,60$, a proporção de insuficiência renal no grupo com sucesso $(2,9 \%)$ foi significativamente menor do que no grupo com insucesso (30,8\%), com $p=0,003$.

Foram estudados vários modelos (Tabelas 3, 4 e 5) selecionados pelo método de regressão logística a partir das variáveis de mecânica pulmonar, laboratorial, gasometria, clínica e unidas (todas juntas), respectivamente. O modelo forneceu os parâmetros como coeficiente, erro-padrão e o nível de significância (valor de p) para cada fator prognóstico significativo. O risco relativo (RR) e seu respectivo intervalo de confiança de $95 \%$ para cada fator foram calculados a partir do modelo de regressão. $O$ valor da estatística $C$ foi calculado para o modelo geral, que avalia a associação entre as probabilidades preditas (calculadas pela regressão logística) e as proporções observadas de sucesso. Quanto mais próximo de um for a estatística C, mais forte a associação, o que expressou boa qualidade de ajuste do modelo proposto.

Modelo 1 - Variável dependente: sucesso ao desmame (Tabela 3). 
Tabela 2 - Análise Estatística das Variáveis Numéricas Segundo o Grupo

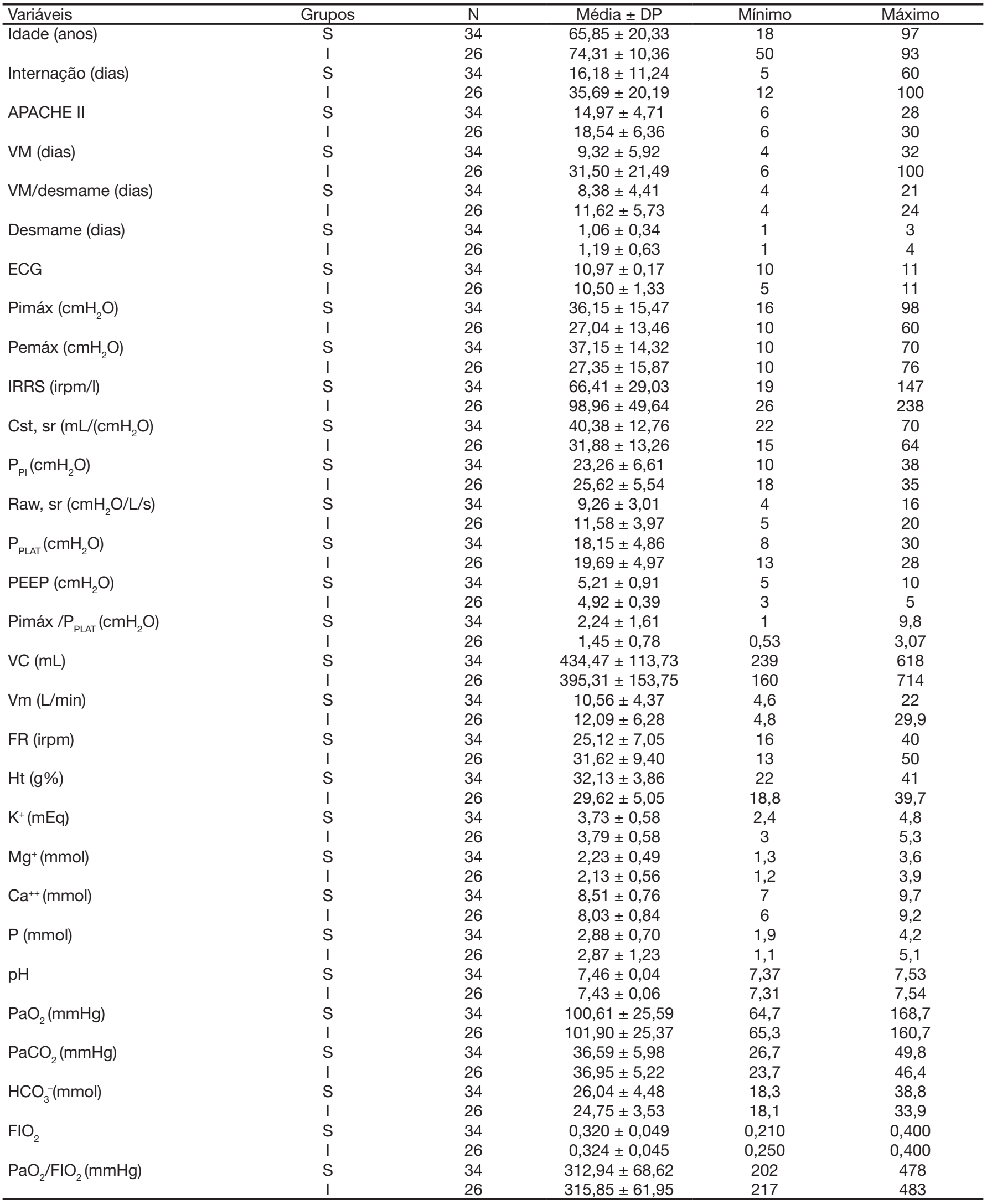

$\mathrm{P}_{\mathrm{PI}-}$ pressão de pico; $\mathrm{P}_{\mathrm{PLAT}-}$ pressão de platô; $\mathrm{VC}$-volume-corrente; Vm-volume-minuto 
AVALIAÇÃO DO SUCESSO DO DESMAME DA VENTILAÇÃO MECÂNICA

Tabela 3 - Resultado da Regressão Logística para Sucesso do Desmame - Modelo 1

\begin{tabular}{ccccccc}
\hline $\begin{array}{c}\text { Fator Prognóstico } \\
\text { Significativo }\end{array}$ & Coeficiente & Erro-Padrão & Valor de p & RR & $\begin{array}{c}\text { Intervalo de } \\
\text { Confiança (95\%) }\end{array}$ & $\begin{array}{c}\text { Estatística C do } \\
\text { Modelo }\end{array}$ \\
\hline & Intercepto & $-1,6023$ & 0,6221 & & & $1,58-20,27$ \\
1 & Pimáx $>(-) 20$ & 1,7327 & 0,6512 & 0,007 & 5,66 & 0,77 \\
2 & IRRS $\leq 78$ & 1,3077 & 0,5992 & 0,029 & 3,70 & $1,14-11,97$ \\
\hline
\end{tabular}

$\mathrm{RR}=$ Risco relativo

Tabela 4 - Resultado da Regressão Logística para o Sucesso do Desmame - Modelo 2

\begin{tabular}{ccccccc}
\hline $\begin{array}{c}\text { Fator Prognóstico } \\
\text { Significativo }\end{array}$ & Coeficiente & Erro-Padrão & $\begin{array}{c}\text { Valor } \\
\text { de } p\end{array}$ & RR & $\begin{array}{c}\text { Intervalo de Con- } \\
\text { fiança (95\%) }\end{array}$ & $\begin{array}{c}\text { Estatística C do } \\
\text { Modelo }\end{array}$ \\
\hline & Intercepto & $-0,4666$ & 0,4865 & & & \\
1 & APACHE $\leq 16$ & 1,7199 & 0,6209 & 0,0004 & 5,58 & $4,37-6,80$ \\
2 & Insuficiência renal & $-2,2169$ & 1,1517 & 0,030 & 0,11 & $-2,15-2,37$ \\
\hline
\end{tabular}

$\mathrm{RR}=$ Risco relativo

Tabela 5 - Resultado da Regressão Logística para o Sucesso do Desmame - Modelo 3

\begin{tabular}{ccccccc}
\hline & $\begin{array}{c}\text { Fator Prognóstico } \\
\text { Significativo }\end{array}$ & Coeficiente & Erro-Padrão & $\begin{array}{c}\text { Valor } \\
\text { de } p\end{array}$ & $\begin{array}{c}\text { RR } \\
\text { Intervalo de Con- } \\
\text { fiança (95\%) }\end{array} \begin{array}{c}\text { Estatística C do } \\
\text { Modelo }\end{array}$ \\
\hline & Intercepto & $-29,695$ & 0,9516 & & & $6,14-8,78$ \\
1 & APACHE $\leq 16$ & 2,0099 & 0,6742 & 0,0004 & 7,46 & $3,89-6,86$ \\
2 & Pimáx $>(-) 20$ & 1,6820 & 0,7563 & 0,006 & 5,38 & 0,82 \\
3 & Dias de VM/desma- & & & & \\
& me $\leq 8$ & 1,4824 & 0,6911 & 0,025 & 4,40 & $3,05-5,76$ \\
\hline
\end{tabular}

$\mathrm{RR}=$ Risco relativo

Analisando simultaneamente os fatores de mecânica pulmonar, segundo a regressão logística, observou-se que a Pimáx $>(-) 20$ e o IRRS $\leq 78$ foram estatisticamente significativos para predizer o sucesso ao desmame, nessa ordem de capacidade explicativa.

Modelo 2 - Variável dependente: sucesso ao desmame (Tabela 4).

Analisando simultaneamente os fatores clínicos, segundo a regressão logística, observou-se que o APACHE II $\leq 16$ e a ausência de insuficiência renal foram estatisticamente significativas para predizer o sucesso do desmame, nessa ordem de capacidade explicativa. Modelo 3 - Variável dependente: sucesso ao desmame (Tabela 5).

Analisando simultaneamente todos os fatores, segundo a regressão logística, no nível de 5\%, observou-se que APACHE II $\leq 16$, Pimáx $>(-) 20$ e dias de VM/desmame $\leq 8$ foram estatisticamente significativos para predizer o sucesso do desmame.

\section{DISCUSSÃO}

A regressão logística realizada para avaliar a influência simultânea de fatores mecânicos e clínicos, o APACHE II $\leq 16$, a Pimáx $>(-) 20 \mathrm{cmH}_{2} \mathrm{O}$ e os dias de VM $\leq$ 8 foram estatisticamente significativos para predizer o sucesso do desmame, nessa ordem de capacidade explicativa. Nos dias que se seguem em ventilação mecânica, o prognóstico do paciente modifica-se, e o APACHE II de internação pode diminuir ou elevar-se de acordo com a evolução ${ }^{11,12}$.

Nestes pacientes, os eletrólitos em média não se alteraram, devido à preocupação da equipe do CTI em monitorar e corrigir diariamente os eletrólitos do pacientes sob ventilação mecânica. No estudo, foi avaliado o valor do hematócrito e sua relação com o sucesso do desmame. O grupo com sucesso no desmame da VM apresentou hematócrito de $32 \pm 3 \mathrm{~g} \%$, equivalendo à hemoglobina de $10,5 \pm 1 \mathrm{~g} \%$ no dia do início do desmame, significativamente maior do que o grupo com insucesso. Um subgrupo de 730 pacientes recebendo ventilação mecânica de um grande estudo canaden$\mathrm{se}^{13}$ sobre transfusão sangüínea em CTI foi dividido em dois grupos: um recebendo transfusão caso a hemoglobina fosse $<7 \mathrm{~g} \%$ e outro se a hemoglobina fosse $<10 \mathrm{~g} \%$. Não houve diferença estatística no desmame dos dois grupos, mas esse estudo não foi projetado primariamente para avaliá-lo e muitos dos pacientes foram desmamados com pouco tempo de VM, o que pode levar a crer que tenham suportado níveis menores de hemoglobina. Estudos demonstraram que, em pacientes com doença pulmonar obstrutiva crônica 
que tinham repetido insucessos, a manutenção da hemoglobina $>12 \mathrm{~g} \%$ levou ao sucesso do desmame em todos os pacientes ${ }^{13-15}$.

Nos resultados apresentados neste trabalho da análise estatística das variáveis categóricas segundo o grupo sucesso e insucesso, observou-se que a proporção de homens no grupo com sucesso $(64,7 \%)$ não difere significativamente do grupo com insucesso (50\%), e não houve diferença estatística significativa quanto ao sexo dos grupos com sucesso e insucesso, o que é compatível com o estudo de Epstein e Vuong ${ }^{16}$ sobre a evolução do desmame e a influência do sexo. Quanto à influência da idade, os trabalhos são controversos, pois os resultados variaram, dependendo da população. A média de idade da população estudada foi de $69,52 \pm 17,16$, praticamente de idosos, com tempo de ventilação mecânica de 18,93 \pm 18,39) e APACHE Il de 16,52 $\pm 5,72$ ). A idade como fator isolado não foi importante para determinar o tempo da ventilação mecânica ${ }^{17}$.

A proporção do motivo da internação no grupo com sucesso, não diferiu significativamente do grupo com insucesso. Os pacientes incluídos no estudo possuíam critérios para desmame bem estabelecidos ou o desmame era decidido pelo seu médico-assistente, independentemente desses critérios. Coincidentemente, todos os pacientes antes de serem desmamados possuíam estabilidade hemodinâmica e ausência de desnutrição. Tinham controle da causa que motivou a ventilação mecânica, ausência de secreção abundante e de febre, de modo que $100 \%$ dos pacientes acabaram por satisfazer os critérios clínicos básicos para o desmame, mesmo com a interferência médico-assistente.

A proporção de pneumonia pré-internação no CTI (comunitária ou nosocomial) no grupo com sucesso $(55,9 \%)$ foi significativamente maior do que no grupo com insucesso (23,1\%). Dezoito pacientes que evoluíram com insucesso apresentaram pneumonia associada à ventilação mecânica (PAVM) por Pseudomonas aeruginosa, totalizando $73 \%$ dos casos e, desses, 11 evoluíram para óbito (61\%). Um estudo prospectivo multicêntrico recente evidenciou que pacientes que são re-intubados após o desmame apresentam maior morbidade, maior probabilidade de morte e também risco maior de desenvolver pneumonia nosocomial ${ }^{18}$. Assim, a pneumonia adquirida antes da ventilação mecânica não prediz um prognóstico ruim para o paciente quanto ao seu desmame, mas a pneumonia associada à ventilação mecânica é causa ou conseqüência do in- sucesso do desmame da prótese respiratória. Observou-se que a proporção do método PSV no grupo com sucesso $(47,1 \%)$ não diferiu significativamente do grupo com insucesso $(53,8 \%)$. O método utilizado para o desmame não influenciou com estatística significativa no tempo e na evolução do desmame. Existem dois grandes estudos sobre desmame. O primeiro realizado por Brochard e col. ${ }^{11}$ concluíram que a PSV estava associada a menor tempo de desmame, quando em comparação com outros métodos. E o segundo por Esteban e col. ${ }^{12}$ concluíram que a taxa de desmame foi melhor com peça T. Resultados significativamente inferiores foram obtidos com ventilação mandatória intermitente sincronizada (SIMV). Ao contrário dos achados do presente trabalho, onde não houve diferenças entre peça T e PSV.

Neste estudo, a proporção de insuficiência renal aguda no grupo com sucesso (2,9\%), foi significativamente menor, do que no grupo com insucesso $(30,8 \%)$, dado que corrobora o fato de que o insucesso está correlacionado com a evolução desfavorável do paciente no CTI, demonstrada pelo desenvolvimento de disfunção orgânica. Tal resultado foi semelhante ao do estudo de Epstein e col. ${ }^{19}$ avaliaram 289 pacientes intubados, sendo que 47 tiveram insucesso e evolução desfavorável com maior risco de morte e permanência prolongada no CTI. A insuficiência renal foi correlacionada como um fator de risco maior de morte e insucesso.

$\mathrm{Na}$ análise de regressão logística realizada para se avaliar a influência simultânea de fatores clínicos, o APA$\mathrm{CHE} \| \leq 16$ e a ausência de insuficiência renal foram estatisticamente significativos para predizer o sucesso do desmame. Esses são índices de gravidade, como demonstrado por Epstein e col. ${ }^{19}$.

$\mathrm{Na}$ análise de regressão logística realizada para se avaliar a influência simultânea dos fatores de mecânica pulmonar, a Pimáx $>$ (-) $20 \mathrm{cmH}_{2} \mathrm{O}$ e o IRRS $\leq 78$ foram estatisticamente significantes para predizer 0 sucesso do desmame, sendo esses índices preditores os mais considerados na atualidade. Para a Pimáx > (-) $20 \mathrm{cmH}_{2} \mathrm{O}$, sensibilidade de 85,29\% e especificidade de $53,85 \%$. A sensibilidade foi compatível com a maioria dos trabalhos (67\% a $100 \%)$; e a especificidade foi superior à da maioria dos trabalhos (7\% a $41 \%)$. Quando a Pimáx é verificada por vários investigadores diferentes e com técnicas inadequadas, as mensurações são subestimadas ${ }^{20}$. A especificidade encontrada nesse protocolo de pesquisa foi superior à da literatura porque as medidas foram realizadas somente por dois investigadores treinados e com a mesma técnica. 
A regressão logística realizada para avaliar a influência simultânea de fatores mecânicos e clínicos, o APACHE II $\leq 16$, a Pimáx $>(-) 20 \mathrm{cmH}_{2} \mathrm{O}$ e os dias de $\mathrm{VM} \leq 8$ antes de iniciar o desmame desses pacientes sob ventilação mecânica predizem o sucesso do desmame. A Pimáx, já citada é uma aferição que depende do investigador e da colaboração do paciente ${ }^{20,21}$, sendo uma variável numérica que contribui para a avaliação clínica da recuperação do paciente. Novos estudos deveriam ser realizados para testar os modelos propostos.

\section{Limitações do Estudo}

O presente estudo foi realizado em CTI de hospital privado com população característica e heterogênea, sendo necessária cautela na sua extrapolação para outros grupos de pacientes.

Estudos devem ser desenvolvidos em cada CTI em particular, para desenvolver modelos próprios .

\section{CONCLUSÕES}

O grupo com sucesso no desmame da VM apresentou APACHE II, dia de VM em que iniciou o desmame, IRRS, Raw, sr e FR menores do que o grupo com insucesso e o grupo com sucesso no desmame da VM apresentou ECG, Pimáx, Pemáx e Cst, sr e Ht maiores do que o grupo com insucesso. A proporção de dias de internação, dias de VM no CTI no grupo com sucesso foi menor do que no grupo com insucesso. APACHE II $\leq 16$, a Pimáx $>(-) 20 \mathrm{cmH}_{2} \mathrm{O}$ e os dias de $\mathrm{VM} \leq 8$ antes de iniciar o desmame desses pacientes sob ventilação mecânica podem predizer o sucesso do desmame.

A monitoração do tempo de VM, a otimização do tratamento no sentido de acelerar o processo de desmame são condutas que visam não só ao sucesso do desmame, mas interferem na evolução e no tempo de internação hospitalar.

\section{REFERÊNCIAS}

01. Chao DC, Scheinhorn DJ - Weaning from mechanical ventilation. Crit Care Clin, 1998;14:799-817.

02. Esteban A, Anzueto A, Alia I et al - How is mechanical ventilation employed in the intensive care unit? An international utilization review. Am J Respir Crit Care Med, 2000;161:1450-1458.

03. Goldwalsser RS - Desmame da Ventilação Mecânica, em: Carvalho CRR - Ventilação Mecânica. SP, RJ, BH: Atheneu. CBMI 2000;8:271-301.

04. Damasceno MPCD, David CDN, Souza PCSP et al - Ventilação mecânica no Brasil. Aspectos epidemiológicos. RBTI, 2006;18:219-228.

05. Chao DC, Scheinhorn DJ, Stearn-Hassenpflug M - Patient-ventilator trigger asynchrony in prolonged mechanical ventilation. Chest, 1997;112:1592-1599.

06. Fernandez R - Timing and Criteria for Beginning Weaning, em: Mancebo $J$, Net A, Brochard L - Mechanical Ventilation and Weaning. Update in Intensive Care Medicine and Emergency Medicine 2002;241-247.

07. Chao DC, Scheinhorn DJ, Stearn-Hassenpflug M - Impact of renal dysfunction on weaning from prolonged mechanical ventilation. Crit Care, 1997;1:101-104.

08. Gluck EH - Predicting eventual success or failure to wean in patients receiving long-term mechanical ventilation. Chest, 1996;110:1018-1024.

09. Neil R. Maclntyre - Evidence-Based Guidelines for Weaning and Discontinuing Ventilatory Support. Chest, 2001;120:375-396.

10. Hebert PC, Blajchman MA, Cook DJ - Do blood transfusions improve outcomes related to mechanical ventilation? Chest, 2001;119:1850-1857.

11. Brochard L, Rauss A, Benito $S$ et al - Comparison of three methods of gradual withdrawal from ventilatory support during weaning from mechanical ventilation. Am J Respir Crit Care Med, 1994;150:896-903.

12. Esteban A, Frutos F, Tobin MJ et al - A comparison of four methods of weaning from mechanical ventilation. N Engl J Med, 1995;332:345-350.

13. Schonhofer B, Bohrer H, Kohler D - Blood transfusion facilitating difficult weaning from the ventilator. Anaesthesia, 1998;53:181-184.

14. Hebert PC, Blajchman MA, Cook DJ - Do blood transfusions improve outcomes related to mechanical ventilation? Chest, 2001;119:1850-1857.

15. Similowski T, Agusti A, MacNee $W$ et al - The potential impact of anaemia of chronic disease in COPD. Eur Respir J, 2006;27:390-396.

16. Epstein SK, Vuong V - Lack of influence of gender on outcomes of mechanically ventilated medical ICU patients. Chest, 1999;116:732-739.

17. Chelluri L, Pinsky MR, Donahoe MP et al - Long-term outcome of critically ill elderly patients requiring intensive care. JAMA, 1993;269:3119-3123.

18. de Lassence A, Alberti C, Azoulay E et al - Impact of unplanned extubation and reintubation after weaning on nosocomial pneumonia risk in the intensive care unit: a prospective multicenter study. Anesthesiology, 2002;97:148-156.

19. Epstein SK, Ciubotaru RL, Wong JB - Effect of failed extubation on the outcome of mechanical ventilation. Chest, 1997;112:186-192.

20. Vallverdu I, Calaf N, Subirana M - Clinical characteristics, respiratory functional parameters, and outcome of two-hour T-piece trial in patients weaning from mechanical ventilation. Am J Respir Crit Care Med, 1998;158:1855-1862.

21. David $\mathrm{CMN}$ - Ventilação Mecânica da Fisiologia à Prática Clínica, Rio de Janeiro: Revinter, 2001. 